\title{
Diffuse Hyperpigmented Subcutaneous Nodules as a Primary Manifestation of Disseminated Bacillus Calmette-Guerin Disease in Young Infants
}

\author{
Sedigheh Rafiei Tabatabaei ${ }^{1}$; Ali Amanati ${ }^{2, *}$; Abdollah Karimi ${ }^{1}$; Kourosh Goudarzipour ${ }^{3}$; \\ Zahra Chavoshzadeh ${ }^{1}$; Maryam Kazemi Aghdam ${ }^{4}$; Mohammad Bagher Haghighi ${ }^{5}$ \\ ${ }_{1}^{1}$ Pediatric Infections Research Center, Mofid Children's Hospital, Shahid Beheshti University of Medical Sciences, Tehran, IR Iran \\ ${ }_{3}^{2}$ Professor Alborzi Clinical Microbiology Research Center, Shiraz University of Medical Sciences, Shiraz, IR Iran \\ ${ }^{3}$ Pediatric Congenital Hemathologic Disorders Research Center, Shahid Beheshti University of Medical Sciences, Tehran, IR Iran \\ ${ }_{5}^{4}$ Pediatric Pathology Research Center, Shahid Beheshti University of Medical Sciences, Tehran, IR Iran \\ ${ }^{5}$ Mofid Children's Hospital, Shahid Beheshti University of Medical Sciences, Tehran, IR Iran \\ ${ }^{*}$ Corresponding author: Ali Amanati, Professor Alborzi Clinical Microbiology Research Center, Shiraz University of Medical Sciences, Shiraz, Iran, Amir Oncology Hospital; Address: \\ Fars - Shiraz - Farhang Shahr - against Kosar pool, Fax: +98-7136325655, Postal code: +98-7187915998, E-mail: ali_amanati_1356@yahoo.com \\ Received: October 21, 2014; Accepted: November 4, 2014
}

\begin{abstract}
Introduction: There are increasing reports of serious adverse events of bacillus Calmette-Guerin (BCG) vaccination in infants with unrecognized primary immunodeficiency disorders (PIDs) in our country. Among these adverse events skin manifestations occur less frequently and are less noticed.

Case Presentation: We report on an 11-months-old boy with prolonged fever and diffuse hyperpigmented subcutaneous nodules. Due to lymphopenia, oral thrush and severe adverse reaction to BCG vaccination, the possibility of primary immunodeficiency was considered for him and immunological investigations were done.

Conclusions: Subcutaneous nodules in the absence of a local reaction at the site of BCG vaccination may be the sole manifestation of disseminated BCG disease.
\end{abstract}

Keywords:Mycobacterium Bovis; Skin; Immunologic Deficiency Syndromes

\section{Introduction}

Disseminated bacillus Calmette-Guerin (BCG) disease has been estimated to occur at a rate of 1.35 per million doses of administered vaccine (varies among different populations) with a high case-fatality rate of 80 to $83 \%$ (14). There are increasing reports of serious adverse events of BCG vaccination in infants with unrecognized primary immunodeficiency disorders (PIDs) in our country (5-12). Reticuloendotelial system (lymphadenopathy and hepatosplenomegaly), blood, bone marrow, lung and osteoarticular involvement are known sites of disseminated BCG disease. Skin manifestations occur less frequently and are less noticed $(3,4,13,14)$. Nodules in the absence of a local reaction at the site of the BCG vaccination may be the sole manifestation of disseminated BCG disease $(2,15)$.

\section{Case Presentation}

An 11-month-old boy was referred to the pediatric infectious ward for assessment of prolonged fever and rash. The patient had been immunized for his age according to the national immunization program. He had a 32-monthold healthy brother. His fever had begun three months before admission. He had no significant signs and symp- toms other than intermittent fever for a few weeks after the primary illness. Few weeks later, diffuse cutaneous lesions appeared primarily in lower extremities. His lesions presented as macules which progressed to subcutaneous nodules (Figure 1).

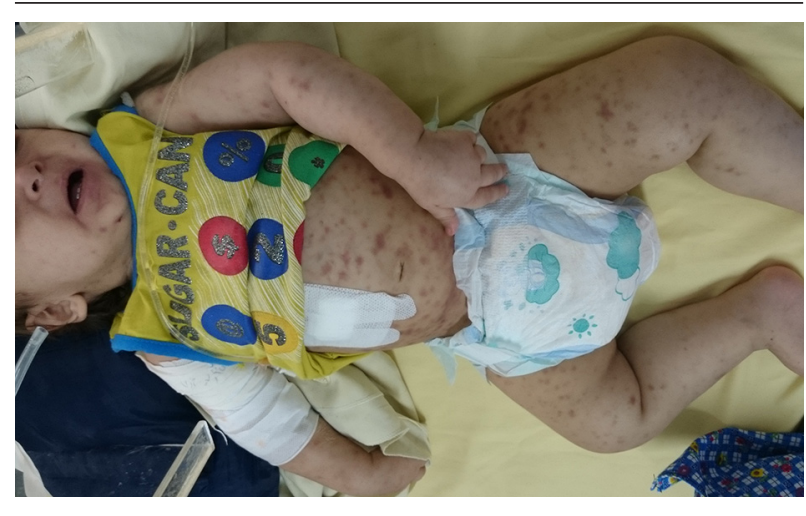

Figure 1. Diffuse Hyperpigmented Subcutaneous Nodules

He was admitted to the local hospital for having fever and rashes. Initial investigations, prior to admission, revealed hypochromic microcytic anemia, leukopenia,

Copyright (C) 2015 Pediartric Infections Research Center. This is an open-access article distributed under the terms of the Creative Commons Attribution-NonCommercial 4.0 International License (http://creativecommons.org/licenses/by-nc/4.0/) which permits copy and redistribute the material just in noncommercial usages, provided the original work is properly cited. 
Rafiei Tabatabaei S et al.

neutropenia and increase in acute phase reactants, including erythrocyte sedimentation rate (ESR) and Creactive protein (CRP). Further evaluation confirmed leukopenia, neutropenia and increase in ESR and CRP, yet no definite diagnosis was achieved and the patient was referred to our hospital. He was admitted initially to the hematology/oncology ward to rule out malignancy and was then transferred to the infectious ward for further investigations. In primary physical examination he was ill and febrile. He looked pale and edematous. Abdominal distention was notable. Generalized hyperpigmented subcutaneous nodules were seen on his entire body. No significant lymphadenopathy was noted. Detection of hepatosplenomegaly was impossible because of abdominal distension. Hemoglobin electrophoresis was normal. Conventional blood culture was negative. Bone marrow aspiration/biopsy was performed without conclusive results. In bone survey, no evidence of lytic or sclerotic lesions was seen except for diffuse subcutaneous masses. Punch biopsy from skin nodules was also performed. Pathological examination revealed non-necrotizing granulomatous inflammation. In Ziehl-Neelsen staining many acid fast bacilli were seen (Figure 2).

Immune status work up was also performed and the possibility of primary immunodeficiency was considered. The flow cytometry immunophenotyping analysis of peripheral blood has been summarized in Table 2. In the immunoglobulin profile study only a low level of IgA was noted. Nitroblue-tetrazolium (NBT) test was also done which was more than 95\%. Early morning gastric washing was performed twice, which was positive for tuberculosis complex-PCR and acid-fast bacilli both times. No active infiltration of lung fields were reported by the chest x-ray, cardio thoracic ratio was in normal ranges, costophrenic angles were clear and soft tissue and bone densities of the thorax were unremarkable.

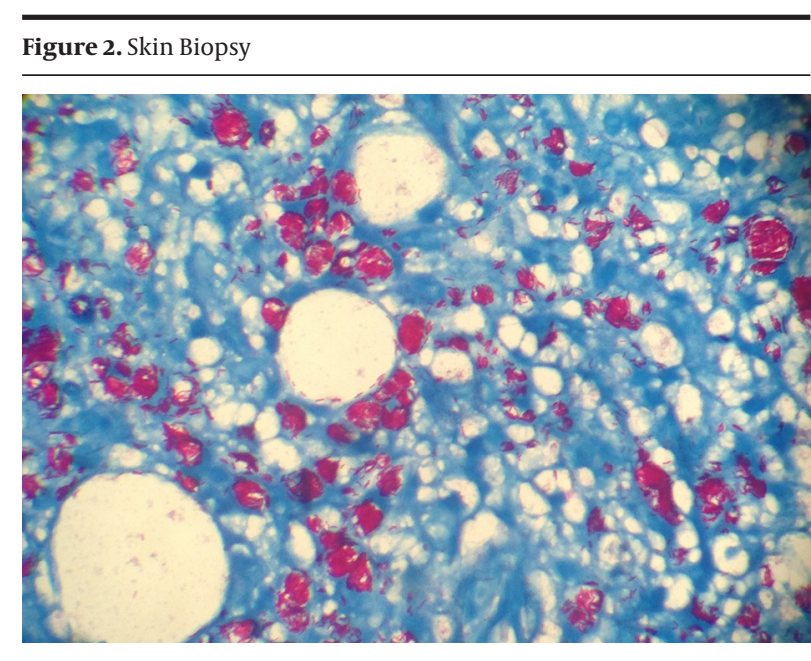

Ziehl-Neelsen staining revealed numerous acid fast bacilli, both intracellularly and extracellularly.

Extensive work was done to rule out other possible viral and parasitic opportunistic infections (Table 1).

Spiral chest computed tomography scan without contrast showed bilateral peribronchial infiltrations with collapse at the right lower and upper lobs. Band shape atelectasis was seen in the left lower lob. Bilateral subpleural ground glass opacities were also seen in dependent parts of both lungs (Figure 3). Anti-mycobacterium bovis treatment was begun with isoniazid, rifampin, ethambutol, ciprofloxacin and clarithromycin. After a week, amikacin was added to his regimen. Two weeks after drug therapy the fever subsided and the nodules became smaller.

\section{Discussion}

Discussion about differential diagnosis of fever and rashes could be found in review articles and guidelines elsewhere. Nonetheless, neoplastic skin manifestation of hematologic malignancy especially Langerhans cell histiocytosis should always be kept in mind.

\begin{tabular}{|c|c|c|c|}
\hline Virus Marker & Antibody Type & Result & Comment \\
\hline \multirow{2}{*}{$\begin{array}{l}\text { Anti-toxoplasma } \\
\text { Antibody }\end{array}$} & $\operatorname{IgG}$ & 24.9 & Reactive \\
\hline & IgM & 0.25 & Non-reactive \\
\hline \multirow[t]{2}{*}{ Anti CMV Antibody } & $\operatorname{IgG}$ & 9.3 & Borderline \\
\hline & IgM & 0.25 & Non-reactive \\
\hline Anti HCV Antibody & IgM & & Non-reactive \\
\hline \multirow{2}{*}{$\begin{array}{l}\text { Anti EBV Antibody } \\
\text { (VCA) }\end{array}$} & IgG & 1 & Non-reactive \\
\hline & IgM & 0.4 & Non-reactive \\
\hline HIV PCR & & & Negative \\
\hline HIV Antibody & & & Negative \\
\hline $\begin{array}{l}\text { Anti leishmania Anti- } \\
\text { body (IFA) }\end{array}$ & & & Negative \\
\hline
\end{tabular}

\footnotetext{
a Abbreviations: CMV, cytomegalovirus; EBV, Epstein-Barr virus; $\mathrm{HCV}$, hepatitis $\mathrm{C}$ virus; HIV, human immunodeficiency virus; IFA, indirect immunofluorescent-antibody test.
}

Table 2. Flow Cytometry Immunophenotyping Analysis of Peripheral Blood

\begin{tabular}{lccc}
\hline Markers & Patient results (\%) & Absolute Count & Gate \\
\hline CD3 & 3 & $<1$ & Lymphocyte \\
CD4 & $<1$ & $<1$ & Lymphocyte \\
CD8 & 3 & $<1$ & Lymphocyte \\
CD19 & 68 & $<1$ & Lymphocyte \\
CD20 & 61 & $<1$ & Lymphocyte \\
CD16 & 18 & $<1$ & Lymphocyte \\
CD56 & 2 & $<1$ & Lymphocyte \\
CD4/CD8 & 0.33 & & Lymphocyte \\
\hline
\end{tabular}



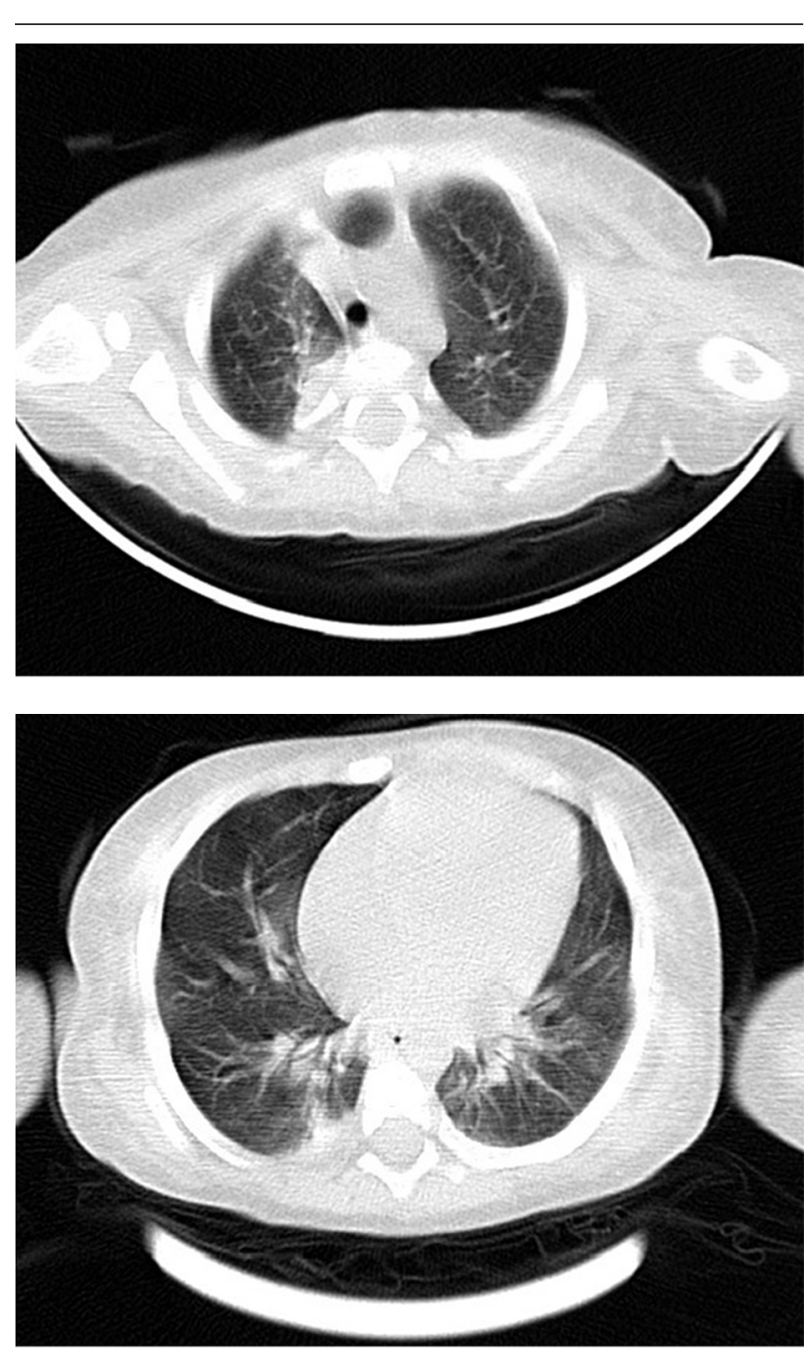

Figure 3. Spiral Chest Computed Tomography Scan Without Contrast Showed Bilateral Subpleural Ground Glass Opacities in Dependent Parts of Both Lungs

These manifestations together with hepatosplenomegaly can closely mimic those of hematological malignancies (16). Our aim for the present report however, was to emphasize on rare manifestation of BCG adverse events in immunocompromised infants with PID. Pediatricians should be mindful of the fact that these complications are not uncommon in immunodeficient patients in our region (5-12). Notably, presence of acid fast bacilli within the cytoplasm (intracellularly) is an uncommon yet interesting phenomena (16). Our patient fulfilled all diagnostic criteria of disseminated BCG infection, including presence Mycobacterium bovis BCG substrain on his skin as indicated by PCR, showing typical histopathological changes with granulomatous inflammation and positive tuberculosis complex-PCR in gastric washing, having systemic symptoms and more than two areas of involvement beyond the site of BCG vaccination (hepatosplenomegaly, pulmonary and skin involvement) $(3,17)$. Patients with unrecognized primary immunodeficiency frequently experience severe complications of live bacterial or viral vaccines (for example BCG and rotavirus) in early infancy. Unusual vaccine adverse events could be the first signs of primary immune deficiencies $(2,3,8,12,17)$; some authors have estimated the incidence of primary immune deficiencies from unusual systemic adverse events of live vaccines in early infancy (2). These complications may partly be the consequence of lack of standard newborn screening programs for primary immunodeficiency. Additionally, inattention to dubious infant death in the family history may also contribute to the late diagnosis and treatment of primary illness. Skin lesions like diffuse hyperpigmented maculopapular rash and subcutaneous nodules could be the primary manifestations of disseminated BCG disease in patients with PID. The cumulative incidence of PID according to a more recent report of Iranian Primary Immunodeficiency Registry (IPIDR) is about 9.7 per 1,000,000 individuals during the last seven years. Among different types of PID, severe combined immunodeficiency was the most common specific disorder (in about 21.1\%; consisting of 154 cases from a total of 731 PID patients). This means a ratio of about one fifth from the total cases of PID who are at increased risk for serious adverse events of BCG vaccination at birth. In fact, more than one third of patients with PID should be considered susceptible to BCG because of susceptibility of infants and children with congenital phagocytic disorder (with prevalence of about 17.4\%) to develop systemic BCG disease (18). According to this report systemic BCG disease was found in 17 cases (2.3 $\%$ ) as the first presentation of PID, during the last seven years (2006 to 2013) (18). Considering the numerous recent reports of serious systemic adverse events of BCG vaccination $(5,7,10-12,19-21)$ and also based on experience in our referral tertiary center, the probability of greater incidence of these side effects as a result of primary immune deficiency comes to mind. Thus, an additional local surveillance system designed to evaluate serious adverse events after live viral and bacterial vaccines at infection wards may be helpful to establish a databank and develop research protocols for clinical evaluation, diagnosis, and management of these adverse events in infants with PID in Iran.

\section{Acknowledgements}

The authors would like to acknowledge the assistance of the infectious, pathology and hematology/oncology ward's staff.

\section{Authors' Contributions}

Abdollah Karimi and Sedigheh Rafiei Tabatabaei designed the study. Mohammad Bagher Haghighi contributed to the acquisition of data. Ali Amanati performed the data interpretation and drafted the manuscript. 
Kourosh Goudarzipour contributed to the hematological work. Maryam Kazemi Aghdam contributed to the interpretation of pathology. Zahra chavoshzadeh contributed to the immunologic work.

\section{References}

1. The role of BCG vaccine in the prevention and control of tuberculosis in the United States. A joint statement by the Advisory Council for the Elimination of Tuberculosis and the Advisory Committee on Immunization Practices. MMWR Recomm Rep. 1996;45(RR-4):1-18.

2. Gonzalez B, Moreno S, Burdach R, Valenzuela MT, Henriquez A, Ramos MI, et al. Clinical presentation of Bacillus CalmetteGuerin infections in patients with immunodeficiency syndromes. Pediatr Infect Dis J.1989;8(4):201-6.

3. Talbot EA, Perkins MD, Silva SF, Frothingham R. Disseminated bacille Calmette-Guerin disease after vaccination: case report and review. Clin Infect Dis. 1997;24(6):1139-46.

4. Toida I, Nakata S. [Severe adverse reactions after vaccination with Japanese BCG vaccine: a review]. Kekkaku. 2007;82(11):809-24.

5. Afshar Paiman S, Siadati A, Mamishi S, Tabatabaie P, Khotaee G. Disseminated Mycobacterium bovis infection after BCG vaccination. Iran J Allergy Asthma Immunol. 2006;5(3):133-7.

6. Farhoudi A, Bazargan N, Arshi S, Pourpak Z. BCG dissemination in 40 patients and review of leukocyte mycobactericidal defect in one patient. Iran J Allergy Asthma Immunol. 2000;1(2):63-7.

7. Norouzi S, Aghamohammadi A, Mamishi S, Rosenzweig SD Rezaei N. Bacillus Calmette-Guerin (BCG) complications associated with primary immunodeficiency diseases. I Infect. 2012;64(6):543-54.

8. Paiman SA, Siadati A, Mamishi S, Tabatabaie P, Khotaee G. Disseminated Mycobacterium bovis infection after BCG vaccination. Iran J Allergy Asthma Immunol. 2006;5(3):133-7.

9. Rezai MS, Khotaei G, Mamishi S, Kheirkhah M, Parvaneh N. Disseminated Bacillus Calmette-Guerin infection after BCG vaccination.J Jrop Pediatr. 2008;54(6):413-6.

10. Sadeghi-Shabestari M, Rezaei N. Disseminated bacille Calmette-
Guerin in Iranian children with severe combined immunodeficiency. Int J Infect Dis. 2009;13(6):e420-3.

11. Sadeghi-Shanbestari M, Ansarin K, Maljaei SH, Rafeey M, Pezeshki Z, Kousha A, et al. Immunologic aspects of patients with disseminated bacille Calmette-Guerin disease in north-west of Iran. Ital J Pediatr. 2009;35:42.

12. Sedighi I, Amanati A, Borzouei B. France: 2010. Disseminated BCG infection due to BCG Vaccination.

13. Al-Herz W, Nanda A. Skin manifestations in primary immunodeficient children. Pediatr Dermatol. 2011;28(5):494-501.

14. Antaya RJ, Gardner ES, Bettencourt MS, Daines M, Denise Y, Uthaisangsook $S$, et al. Cutaneous complications of BCG vaccination in infants with immune disorders: two cases and a review of the literature. Pediatr Dermatol. 2001;18(3):205-9.

15. Abramowsky C, Gonzalez B, Sorensen RU. Disseminated bacillus Calmette-Guerin infections in patients with primary immunodeficiencies. Am J Clin Pathol.1993;100(1):52-6.

16. Gupta N, Kumar V, Nijhawan R, Srinivasan R, Rajwanshi A. FNAC of Bacillus- Calmette- Guerin lymphadenitis masquerading as Langerhans cell histiocytosis. Cytojournal. 2004;1(1):6.

17. Santos A, Dias A, Cordeiro A, Cordinha C, Lemos S, Rocha G, et al. Severe axillary lymphadenitis after BCG vaccination: alert for primary immunodeficiencies. J Microbiol Immunol Infect. 2010;43(6):530-7.

18. Aghamohammadi A, Mohammadinejad P, Abolhassani $\mathrm{H}$ Mirminachi B, Movahedi M, Gharagozlou M, et al. Primary immunodeficiency disorders in Iran: update and new insights from the third report of the national registry. J Clin Immunol. 2014;34(4):478-90.

19. Karimi A, Tabatabaei SR, Amanati A, Ghoroubi J, Karami M. Multifocal Dactylitis as a Consequence of Bacillus CalmetteGuérin Vaccination in a Patient With Severe Combined Immunodeficiency: A Case Report. Arch Pediatr. 2014;3(3):e18526.

20. Jadali F, Karimi A, Armin S, Gharib A, Fallah F, Sharifian M. Detection of Disseminated Mycobactrial Infection Due to BCG Vaccination (BCGosis) in 4 Suspected Infant Autopsies by PCR Method. Iranian J Patholog. 2007;2(3):89-93.

21. Shirvani F, Chavoshzadeh Z, Arjmand R, Karimi A. Four-MonthOld Boy With Fever, Hepatosplenomegaly and Diffuse Pulmonary Infiltrations. Arch Clin Infec Dis. 2014;7(2) 